\title{
Transcriptional cofactor Mask2 is required for YAP-induced cell growth and migration in bladder cancer cell
}

\author{
Liang Dong*, Fan Lin*, Wanjun Wu, Weiren Huang ${ }^{\bowtie}$, Zhiming Cai ${ }^{\bowtie}$ \\ State Engineering Laboratory of Medical Key Technologies Application of Synthetic Biology, Shenzhen Second People's Hospital, The First Affiliated Hospital of \\ Shenzhen University, Shenzhen 518039, PR China. \\ *These authors contributed equally to this work. \\ $\triangle$ Corresponding authors: Dr. Weiren Huang Email: pony8980@163.com; Prof. Zhiming Cai Email: caizhiming2000@163.com
}

(C) Ivyspring International Publisher. Reproduction is permitted for personal, noncommercial use, provided that the article is in whole, unmodified, and properly cited. See http://ivyspring.com/terms for terms and conditions.

Received: 2016.06.09; Accepted: 2016.09.03; Published: 2016.10.23

\begin{abstract}
The highly conserved Hippo signaling pathway is an important pathway involved in tumorigenesis and development. In previous studies, YAP, the transcription coactivator of Hippo pathway, is found to be highly expressed in many clinical bladder cancer samples. To investigate the function of YAP and its cofactor Mask2 in bladder cancer, we overexpress YAP in bladder cancer cells and discover that YAP is able to promote bladder cancer cell growth and migration. In addition, we provide evidence that knockdown of Mask2 is able to repress bladder cancer cell growth and migration. Furthermore, we demonstrate knockdown of Mask2 is able to inhibit bladder cancer cell growth and migration induced by the excessive YAP. To explain the function of YAP/Mask2 complex in bladder cancer, we check the target genes' expression of Hippo signaling pathway involved in cell growth and migration and find overexpressed YAP is able to upregulate the target genes' expression while depletion of Mask2 downregulates them. Taken together, we demonstrate that Mask2 is required for the function of bladder cancer cell growth and migration induced by YAP via the target genes of Hippo pathway.
\end{abstract}

Key words: Mask2; YAP; cell growth and migration; bladder cancer

\section{Introduction}

Bladder cancer is one of the most common types of malignancies worldwide and frequently causes death in patients with advanced-stage cancer. Effective therapeutic targets for tumorigenesis and progression of bladder cancer are still poorly known $[1,2]$.

Currently, several pathways have been uncovered to regulate bladder cancer cell growth and migration through different mechanism, such as mTOR, EGFR, JAK-STAT, apoptosis pathways [3]. However, the function of evolutionarily conserved Hippo pathway, which plays an important role in cell growth and migration, wasn't well understood during tumorigenesis and progression of bladder cancer. The Hippo pathway acts via the Yes-associated protein (YAP) transcriptional coactivator to control cell growth and migration in mammals [4, 5]. YAP is a potential oncogene that is up-regulated in diverse cancers. Previous studies showed YAP was highly expressed in bladder cancer tissues and the expression level of YAP was critical for bladder cancer cell growth and migration [6-10]. Recently a novel cofactor of YAP, named Mask1/2, was identified. They interacted with YAP to regulate Hippo pathway [11, 12]. Mask1 (or ANKHD1) played an important role in cancer cell growth, such as prostate cancer, myeloma and leukemia cells [13-18]. However, the cellular behavior or mechanisms of 
Mask2 (or ANKRD17) in cancer cells was unknown, although Mask2 was reported to involve in immune signaling [19].

In this study, we demonstrated that YAP was up-regulated in some bladder cancer cell (5637). Overexpressed YAP promoted bladder cancer cell growth and migration while silencing Mask2 inhibited these processes induced by YAP. Furthermore, YAP/Mask2 complex regulated the expression of the target genes in Hippo pathway to control bladder cancer cell growth and migration. Our findings clarify the potential function and mechanism of Mask2 in bladder cancer cell, offering essential scientific basis for new targets in bladder cancer.

\section{Results and discussion}

\section{Elevated expression of YAP in 5637 cells promotes cell growth and migration}

To study the function of YAP in bladder cancer cells, we first detected YAP expression level in normal urothelial cell (SV-HUC-1) and some bladder cancer cell lines (T24, 5637, UMUC-3 and SW-780) via qPCR assay. As Figure 1A showed, YAP was highly expressed in 5637 cell, but not in normal urothelial cell and other bladder cancer cell lines. Therefore, 5637 cell may be one of the best cell models to investigate the function and mechanism of YAP in bladder cancer, especially for YAP highly expressed bladder cancer.
A
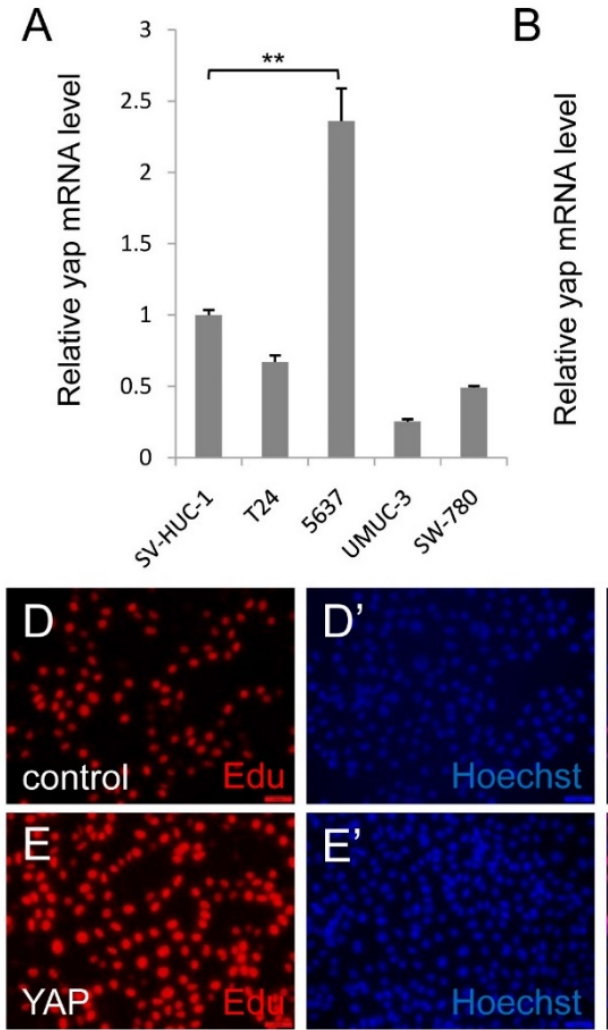

$\mathrm{Oh}$

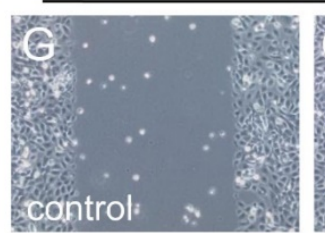

B
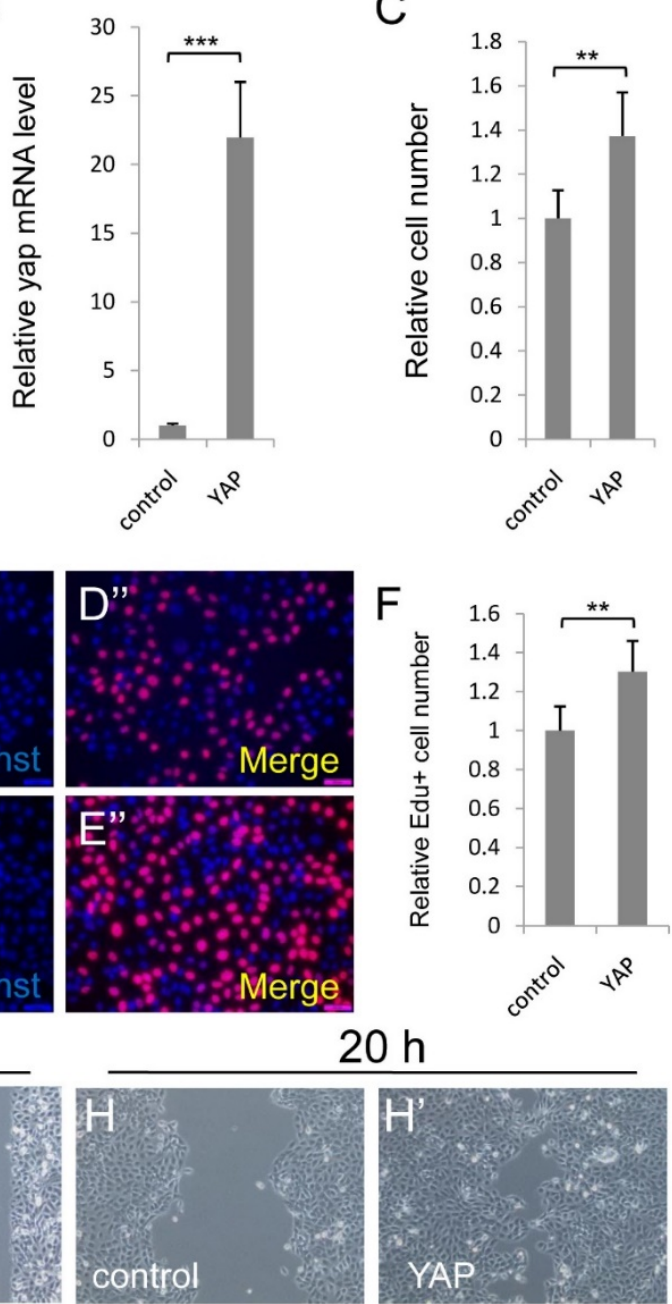

Figure 1. Elevated expression of YAP in $\mathbf{5 6 3 7}$ cells promotes cell growth and migration. (A) qPCR to check YAP expression level in different bladder cancer cells (SV-HUC-1 is normal urothelial cell, T24, 5637, UMUC-3 and SW-780 are different bladder cancer cells); (B) Overexpression of YAP is checked by qPCR in 5637 cell; (C) 5637 cell growth is measured by CCK assay; (D-F) 5637 cell proliferation is measured by Edu staining assay; (G-H) 5637 cell migration is detected. (5637 cells were transfected with control or YAP plasmid. All data are shown as mean \pm SD, $* *, p<0.01, * * *, p<0.001)$ 
To investigate the function of upregulated YAP in bladder cancer, we overexpressed YAP in 5637 cell, and performed CCK-8 assay to check the cell growth effect. More cells were detected by the assay, when YAP was overexpressed (Figure $1 \mathrm{~B}$ and $1 \mathrm{C}$ ). To confirm the finding, we performed the Edu staining assay to check the cell proliferation effect of YAP. Consistently, overexpression of YAP induced the bladder cancer cell proliferation (Figure 1D-1F). Furthermore, overexpressed YAP significantly promoted 5637 migrations by wound-healing assay (Figure $1 \mathrm{G}$ and $1 \mathrm{H}$ ). Taken together, these data suggested that overexpressed YAP in 5637 cells promotes cell growth and migration.

\section{Depletion of Mask2 inhibits cell growth and migration in bladder cancer cell}

Mask2, the novel cofactor of YAP, has been identified recently. However, its function in tumorigenesis and development is still unknown. To dissect the function of Mask2 in bladder cancer, we firstly checked Mask2 expression level and discovered Mask2 expressed higher in 5637 cell than other bladder cancer cell lines (T24, UMUC-3 and SW-780) (Figure S1). Moreover, we knocked down Mask2 in 5637 cell and found depletion of Mask2 reduced the cell growth in the CCK-8 assay (Figure 2B) and the RNAi efficiency of shMask2 was checked by qPCR (Figure 2A). To confirm the cell growth effect, we performed the Edu staining assay to check the effect of Mask2 on 5637 cell proliferation. Consistent with previous data, Mask2 RNAi reduced the bladder cancer cell proliferation (Figure 2C-E). Moreover, knockdown of Mask2 reduced 5637 migrations by wound-healing assay (compared Figure 3G and 3G" with $3 \mathrm{~F}$ and $\left.3 \mathrm{~F}^{\prime \prime}\right)$. Collectively, these data indicated that depletion of Mask2 inhibits cell growth and migration in 5637 cell.
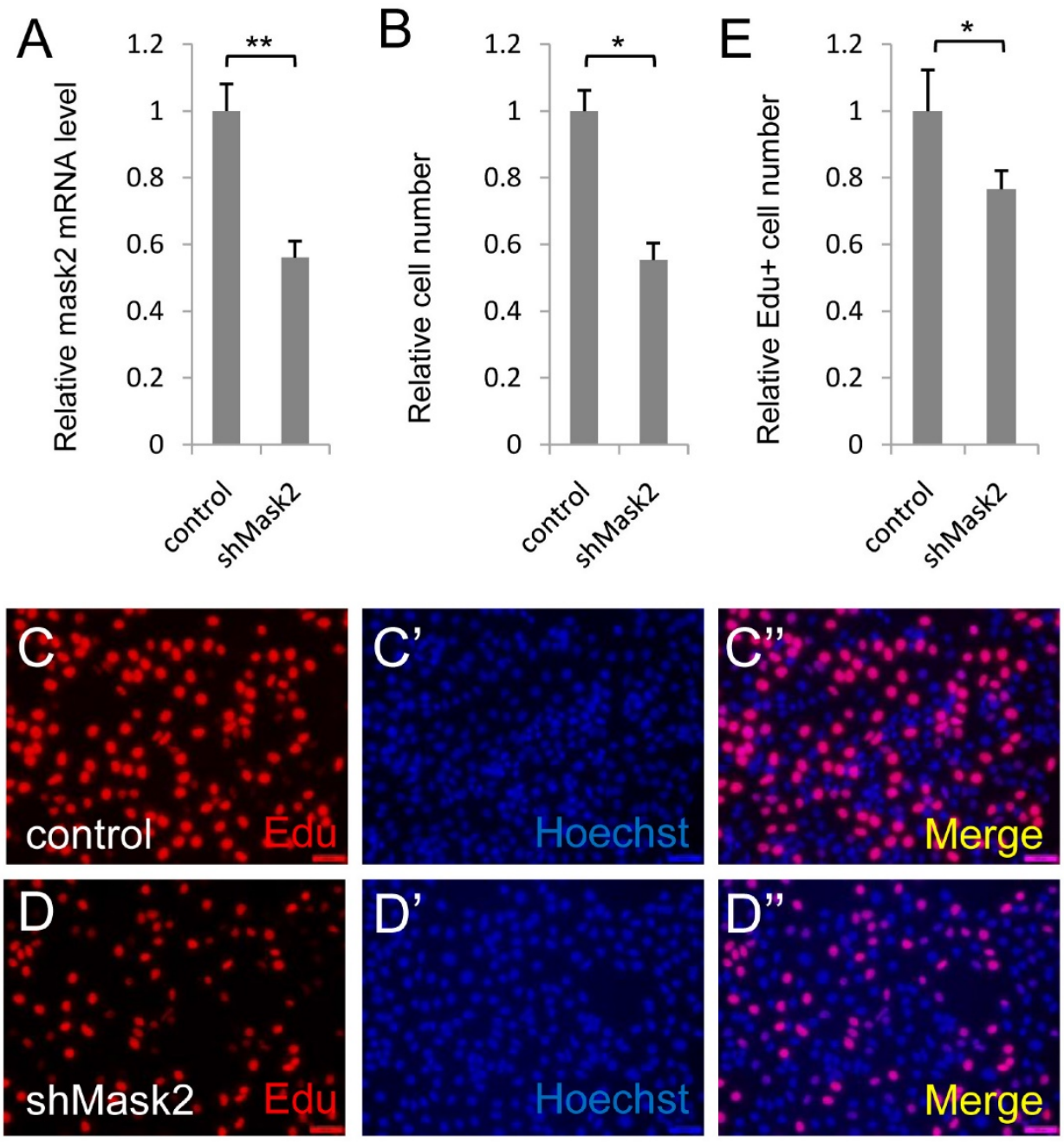

Figure 2. Depletion of Mask2 inhibits cell growth in bladder cancer cell. (A) Knockdown of Mask2 is checked by qPCR in 5637 cell; (B) 5637 cell growth is measured by CCK assay; (C-E) 5637 cell proliferation is measured by Edu staining assay. (5637 cells were transfected with control or shMask2 plasmid. All data are shown as mean $\pm \mathrm{SD}, *, p<0.05, * *, p<0.01)$ 

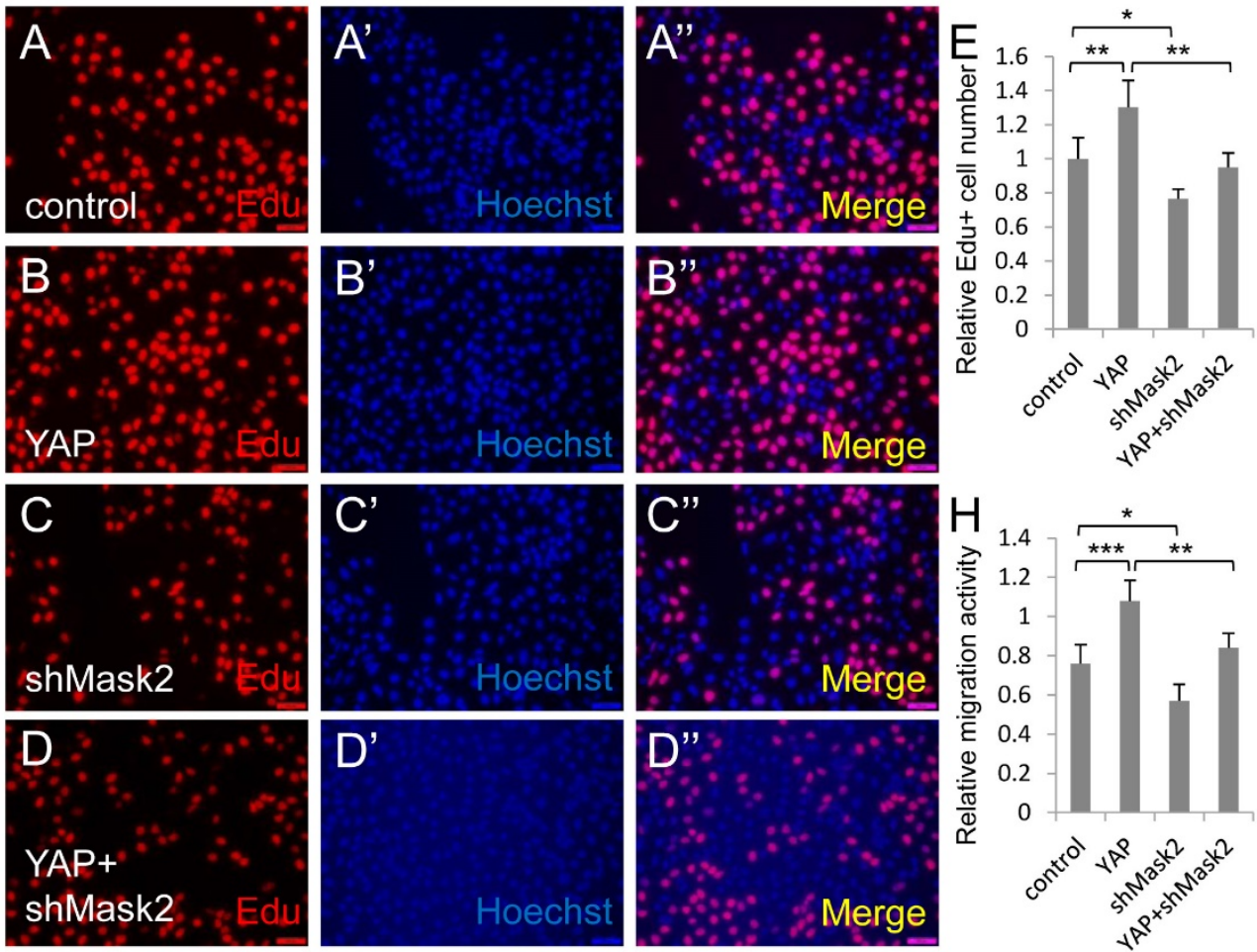

$\mathrm{Oh}$
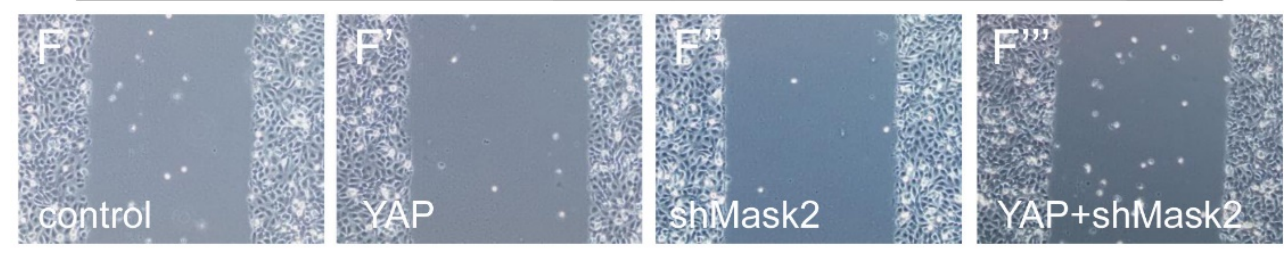

$20 \mathrm{~h}$
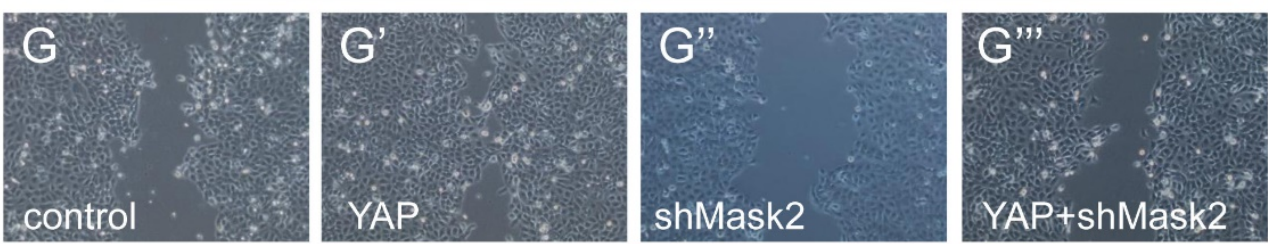

Figure 3. Mask2 is required for YAP induced bladder cancer cell growth and migration. (A-E) 5637 cell proliferation is measured by Edu staining assay; (F-H) 5637 cell migration is detected by wound healing assay. (5637 cells were respectively transfected with control, YAP, shMask2 or YAP plus shMask2 plasmids. All data are shown as mean \pm SD, *, $p<0.05, * *, p<0.01, * * *, p<0.001)$

\section{Mask2 is required for YAP induced bladder cancer cell growth and migration}

To gain insight into the relationship between Mask2 and YAP during bladder cancer tumorigenesis and development, we co-expressed YAP and shMask2 in 5637, via Edu staining assay, and find that depletion of Mask2 was able to obviously abolish YAP induced bladder cancer cell overgrowth (Figure 3A-3E). Similarly, knockdown of Mask2 was able to efficiently inhibit the 5637 cell migration caused by YAP overexpression (Figure 3F-3H). Taken all together, these results suggested that Mask2 is required for YAP induced bladder cancer cell growth and migration. This finding may provide evidence that Mask2 may be a potential therapy target for YAP highly expressed bladder cancer.

\section{YAP/Mask2 transcriptional complex regulates the expression of the Hippo pathway target genes}

Finally, we detected the expression of the target genes in this pathway (CTGF and cyr61) by qPCR assay, which play critical role in cell growth and migration. It was found YAP overexpression upregulated the target genes' expression, meanwhile Mask2 RNAi reduced them in 5637 cell (Figure 4A-D). Collectively, we proposed a potential working model 
that Mask2 controlled the expression of the Hippo pathway's target genes (CTGF, cyr61, et al) to affect bladder cancer cell growth and migration via forming a complex with YAP. Thus Mask2 is required for YAP overexpression induced bladder cancer cell growth and migration by regulating the same target genes, which may offer a potential novel target of bladder cancer therapy (Figure 4E). Our discovery would give a new application basis of Mask2 in cancer treatment, especially for YAP highly expressed bladder cancer.

\section{Conclusion}

This study demonstrated that YAP was up-regulated in some bladder cancer cell (5637) and overexpressed YAP promoted bladder cancer cell growth and migration. Mask2 is required for YAP induced bladder cancer cell growth and migration. YAP/Mask2 regulates the expression of the target genes in Hippo pathway to control bladder cancer cell growth and migration.

\section{Materials and methods}

\section{Plasmids construction}

shMask2 plasmid with the target sequence "TGCTAACTTTAACAGACAA" was purchased from genechem company (Shanghai, China). YAP plasmid was purchased from Addgene (ID: 42555).

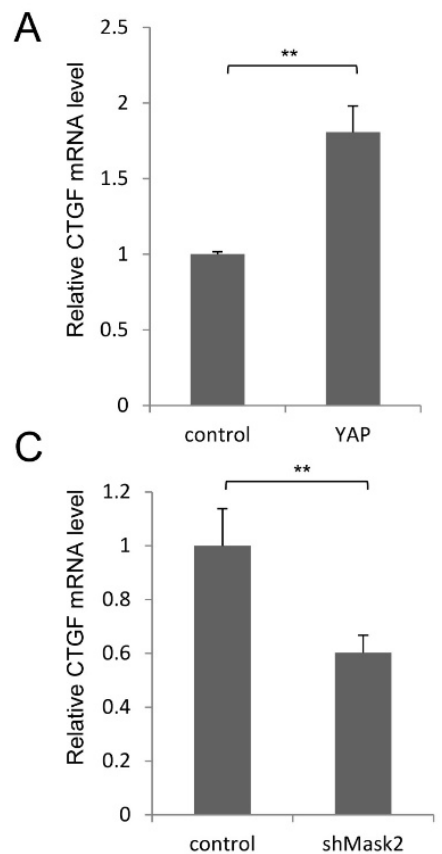

B
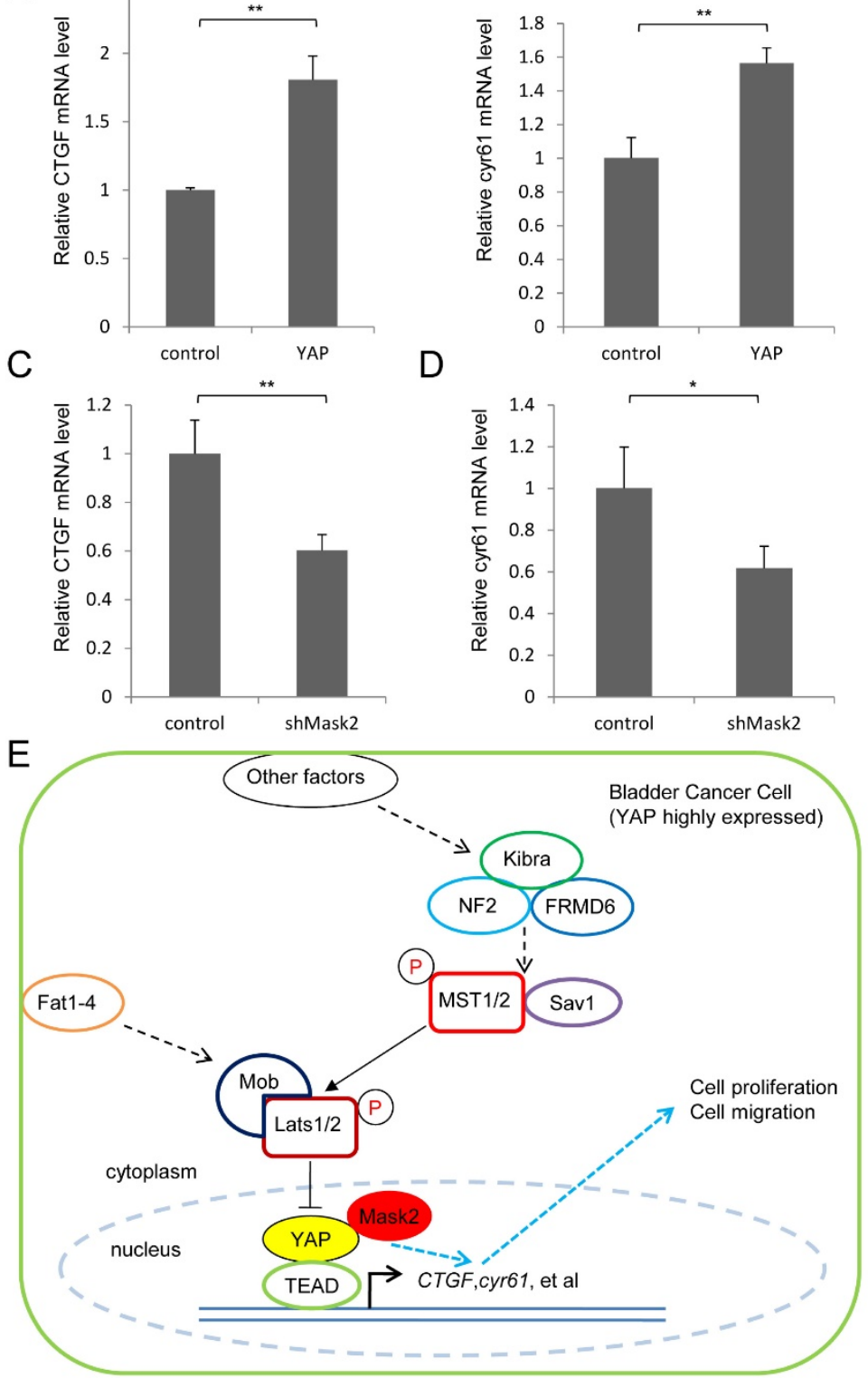

Figure 4. YAP/Mask2 transcriptional complex regulate the expression of the Hippo pathway target genes. (A-D) qPCR to check the expression level of target genes (CTGF and cyr6I) in the Hippo pathway in 5637 cell; (E) Working model of Mask2 in bladder cancer cell (YAP highly expressed): Mask2, as the cofactor of YAP, regulates the expression of the Hippo pathway's target genes (CTGF, cyr6l, et al) to control bladder cancer cell growth and migration, thus is required for YAP overexpression induced bladder cancer cell growth and migration. (All data are shown as mean \pm SD, $*, p<0.05, * *, p<0.01$ ) 


\section{Cell culture and transfections}

Human normal immortal urothelial cell (SV-HUC-1), bladder cancer cells (5637, UMUC-3, T24 and SW-780) were purchased from SIBS, CAS, Shanghai, China. The SV-HUC-1 cell was cultured in F-12K medium. The 5637, UMUC-3, T24, and SW-780 cells were maintained in DMEM media or RPMI-1640 media, respectively. All cells were grown at $37{ }^{\circ} \mathrm{C}$ in an atmosphere of $5 \% \mathrm{CO}_{2}$.

For all transfection experiments, cells were transfected with Lipofactamine 3000 (Invitrogen) following the manufacture's protocols.

\section{Quantitative Real-Time PCR Analysis}

Total RNAs from cells were isolated using the Trizol reagent (Invitrogen) following the manufacture's instruction. cDNA was synthesized by SuperScript III ${ }^{\circledR}$ (Invitrogene) kit. Quantitative real-time PCR (qPCR) was performed on the ABI PRISM 7000 Fluorescent Quantitative PCR system (Applied Biosystems) with SYBR Green Premix (Takara). All samples were normalized to gapdh and the relative mRNA levels were calculated by $\Delta \Delta \mathrm{Ct}$ method. The primers used (from $5^{\prime}$ to $3^{\prime}$ direction) were listed as following:

gapdh F: TCATCCCTGCCTCTACTG;

gapdh R: TGCTTCACCACCTTCTTG;

yap F: GGAATTGAGAACAATGACGA;

yap R: TAGTATCACCTGTATCCATCTC;

mask2 F: GCAGATAACCGCAAGATAACTC;

mask2 R: CTTCTTCAGCATCTCCTTATCAG;

CTGF F: CCAATGACAACGCCTCCTG;

CTGF R: TGGTGCAGCCAGAAAGCTC; cyr61 F: AGCCTCGCATCCTATACAACC;

cyr61 R: TTCTTTCACAAGGCGGCACTC.

\section{CCK-8 assay}

The effects of YAP/Mask2 complex on cell growth were determined by Cell Counting Kit-8 assay (Transgene, China). Briefly, $5 \times 10^{3}$ cells per well were seeded in a 96-well plate for 12 hours and then transfected as mentioned above. After 48 hours of transfection, $100 \mu \mathrm{l}$ fresh medium containing $10 \%$ of CCK-8 was replaced to each well and the cells were cultured for another 1 hour. The absorbance at $450 \mathrm{~nm}$ was determined using an ELISA microplate reader (Bio-Rad). Experiments were repeated for three times.

\section{Edu incorporation assay}

The effects of YAP and shMask2 on cell proliferation were determined by Ethynyl-2deoxyuridine incorporation assay using Cell-Light ${ }^{\mathrm{TM}}$ EdU Apollo®567 In Vitro Imaging Kit (Ribobio, China). Briefly, after transfection for 48 hours, the Edu was added to each well with a final concentration of $50 \mu \mathrm{M}$. After 2 hours' culture, cells were fixed with $4 \%$ paraformaldehyde at room temperature. After 3 times washing in PBST (PBS with $0.1 \%$ Triton X-100), cells were incubated with $1 \times$ Apollo solution for $30 \mathrm{~min}$ at room temperature (RT) in the dark. Finally, cells were subjected to nuclear staining with $1 \times$ Hoechst for 30 min and then observed by fluorescence microscopy.

\section{Statistical analysis}

All experiments were performed in triplicate and data were shown as mean \pm standard deviation (SD). Statistical analysis was conducted by Student's t-test or ANOVA using SPSS version 19.0 software. $p<0.05$ was considered statistically significant.

\section{Supplementary Material}

Supplementary tables and figures.

http://www.jcancer.org/v07p2132s1.pdf

\section{Acknowledgement}

This work was supported by the National Key Basic Research Program of China (973 Program) (2014CB745201) and China Postdoctoral Foundation (2015M582462), Shenzhen Municipal Government of China (ZDSYS201504301722174, JCYJ20150330102720 130, ZDSY20130531165409949, GJHZ201503161549124 94), Special Support Funds of Shenzhen for Introduced High-Level Medical Team, and Shenzhen High-Level Medical Discipline Development Program (2016031638).

\section{Competing Interests}

The authors have declared that no competing interest exists.

\section{References}

1. van Kessel KE, Zuiverloon TC, Alberts AR, Boormans JL, Zwarthoff EC. Targeted therapies in bladder cancer: an overview of in vivo research. Nature reviews Urology. 2015

2. Rouanne $\mathrm{M}$, Loriot $\mathrm{Y}$, Lebret $\mathrm{T}$, Soria JC. Novel therapeutic targets in advanced urothelial carcinoma. Critical reviews in oncology/hematology. 2015

3. Abbosh PH, McConkey DJ, Plimack ER. Targeting Signaling Transduction Pathways in Bladder Cancer. Current oncology reports. 2015; 17: 58.

4. Mo JS, Park HW, Guan KL. The Hippo signaling pathway in stem cell biology and cancer. EMBO reports. 2014; 15: 642-56.

5. Dong L, Li J, Huang H, Yin MX, Xu J, Li P, et al. Growth suppressor lingerer regulates bantam microRNA to restrict organ size. Journal of molecular cell biology. 2015

6. Li S, Yu Z, Chen SS, Li F, Lei CY, Chen XX, et al. The YAP1 oncogene contributes to bladder cancer cell proliferation and migration by regulating the H19 long noncoding RNA. Urologic oncology. 2015.

7. Ciamporcero E, Shen H, Ramakrishnan S, Yu Ku S, Chintala S, Shen L, et al. YAP activation protects urothelial cell carcinoma from treatment-induced DNA damage. Oncogene. 2015.

8. Gao Y, Shi Q, Xu S, Du C, Liang L, Wu K, et al. Curcumin promotes KLF5 proteasome degradation through downregulating YAP/TAZ in bladder cancer cells. International journal of molecular sciences. 2014; 15: 15173-87.

9. Liu JY, Li YH, Lin HX, Liao YJ, Mai SJ, Liu ZW, et al. Overexpression of YAP1 contributes to progressive features and poor prognosis of human urothelial carcinoma of the bladder. BMC cancer. 2013; 13: 349. 
10. Zhang $\mathrm{L}$, Ren F, Zhang $\mathrm{Q}$, Chen $\mathrm{Y}$, Wang B, Jiang J. The TEAD/TEF family of transcription factor Scalloped mediates Hippo signaling in organ size control. Developmental cell. 2008; 14: 377-87.

11. Sidor CM, Brain R, Thompson BJ. Mask proteins are cofactors of Yorkie/YAP in the Hippo pathway. Current biology : CB. 2013; 23: 223-8.

12. Sansores-Garcia L, Atkins M, Moya IM, Shahmoradgoli M, Tao C, Mills GB, et al. Mask is required for the activity of the Hippo pathway effector Yki/YAP. Current biology : CB. 2013; 23: 229-35.

13. Machado-Neto JA, Lazarini M, Favaro P, de Melo Campos P, Scopim-Ribeiro R, Franchi Junior GC, et al. ANKHD1 silencing inhibits Stathmin 1 activity, cell proliferation and migration of leukemia cells. Biochimica et biophysica acta. 2015; 1853: 583-93.

14. Dhyani A, Machado-Neto JA, Favaro P, Saad ST. ANKHD1 represses p21 (WAF1/CIP1) promoter and promotes multiple myeloma cell growth. European journal of cancer. 2015; 51: 252-9.

15. Machado-Neto JA, Lazarini M, Favaro P, Franchi GC, Jr., Nowill AE, Saad ST, et al. ANKHD1, a novel component of the Hippo signaling pathway, promotes YAP1 activation and cell cycle progression in prostate cancer cells. Experimental cell research. 2014; 324: 137-45.

16. Dhyani A, Duarte AS, Machado-Neto JA, Favaro P, Ortega MM, Olalla Saad ST. ANKHD1 regulates cell cycle progression and proliferation in multiple myeloma cells. FEBS letters. 2012; 586: 4311-8.

17. Traina F, Favaro PM, Medina Sde S, Duarte Ada S, Winnischofer SM, Costa FF, et al. ANKHD1, ankyrin repeat and KH domain containing 1, is overexpressed in acute leukemias and is associated with SHP2 in K562 cells. Biochimica et biophysica acta. 2006; 1762: 828-34.

18. Miles MC, Janket ML, Wheeler ED, Chattopadhyay A, Majumder B, Dericco J, et al. Molecular and functional characterization of a novel splice variant of ANKHD1 that lacks the KH domain and its role in cell survival and apoptosis. The FEBS journal. 2005; 272: 4091-102.

19. Wang $\mathrm{Y}$, Tong X, Li G, Li J, Deng M, Ye X. Ankrd17 positively regulates RIG-I-like receptor (RLR)-mediated immune signaling. European journal of immunology. 2012; 42: 1304-15. 